\title{
Violencia escolar en los centros de Educación Secundaria en Ourense
}

\author{
School violence in Secondary Education centres in Ourense
}

\author{
Dolores Delfina Nieto*, Ana González**, Alba González** \\ *Asesora do Centro de Formación e Recursos (Ourense), **Profesora de Ensino Secundario (Ourense)
}

\begin{abstract}
Resumen
El objetivo del estudio es conocer las posibles manifestaciones de violencia en centros de Educación Secundaria Obligatoria. La muestra quedó compuesta por 1270 alumnos que cursan Educación Secundaria Obligatoria. Para la recogida de datos se ha utilizado el Cuestionario de Violencia Escolar-Revisado (CUVE-R). Los resultados sostienen puntuaciones medias más altas en los factores disrupción en aula $(\mathrm{DA}=2.85)$, y violencia del alumnado $(\mathrm{VA}=2.23)$. Las más bajas se localizan en el factor violencia del profesorado ( $\mathrm{VP}=2.10)$, y en la violencia a través de las tecnologías de la información y comunicación (VTIC $=1.59)$.

Palabras clave: Educación Secundaria Obligatoria, violencia escolar, metodología cuantitativa, adolescentes.
\end{abstract}

\begin{abstract}
The main target of the research is to know the violence manifestations in the Ourense schools from the point of view of the students, with the intention of understanding these situations for contributing to the development of prevention of the violence actions. The sample was composed by 1270 children of the Secondary Education. To collect the information, is used School Violence Questionnaire (CUVE-R). Finally, it is important to say that the highest media punctuations of the four fundamental factors related with the violence are violence inside the class $(\mathrm{DA}=2.85)$ and student violence $(\mathrm{VA}=2.23)$. In contrast, the lowest ones are teacher violence $(\mathrm{VP}=2.10)$ and information and communication technologies (VTIC=1.59).

Keywords Secondary Education, school violence, quantitative method, teenagers.
\end{abstract}

Con el paso de los años, las sociedades se han vuelto cada vez más complejas de tal forma que exigen nuevos patrones de convivencia, basados en una ética de mutuo reconocimiento y un mínimo de competencias cívicas que permitan una convivencia pacífica. En los últimos tiempos, la sociedad española se ha visto sacudida por noticias acerca del incremento de las manifestaciones de violencia en los escenarios escolares. En consecuencia, la enseñanza, universo humano y social desde el que deben afrontarse los grandes retos de la humanidad, debe caminar hacia climas sociales escolares favorables a la convivencia.
Así, la educación es uno de los conceptos más dilatados y con mayor posibilidad de predisponer y gestar una convivencia armónica entre individuos. Las instituciones escolares deben tener siempre presente la necesidad de superar actitudes y comportamientos contrarios a la convivencia pacífica y ejercitarse en la práctica de la misma. Educar para la no violencia en los centros de enseñanza, plantea la necesidad de comprenderla y entrever los procedimientos psicosocioeducativos para afrontarla, lo cual supone aprender a pensar y actuar desde propuestas conflictivas no violentas.

Gran parte de los estudios e investigaciones ocupadas en la temática han establecido una firme dependencia entre la violencia en los centros educativos y las acentuadas mutaciones económicas y sociales experimentadas por las sociedades actuales. En este sentido, las situaciones de violencia experimentadas en las escuelas, pueden responder al desfase que se produce entre las viejas formas de establecimiento del orden y las nuevas formas que no parecen lograr una eficacia imprescindible para regular una convivencia pacífica.

Desde esta perspectiva, los centros educativos han ido introduciendo cambios importantes en el tratamiento de la convivencia y en la resolución de conflictos, con la implicación de las administraciones educativas y la dotación de legislación, protocolos de actuación, espacios, y normativa adaptada a cada centro. No obstante, continúa siendo una asignatura pendiente que debe mejorar, retomando aspectos como la formación personalizada de los docentes, la dotación de recursos económicos, la reducción horaria de los encargados de la convivencia y, sobre todo, el trabajo con las familias.

Esta investigación nace con el propósito de conocer en profundidad algunos aspectos relacionados con la violencia escolar de los adolescentes ourensanos vivenciados en el contexto escolar. Consecuentemente la principal finalidad del estudio es avanzar en la comprensión del fenómeno de la violencia escolar, desde el punto de vista del alumnado, con la única intención de ayudar en el diseño de programas o medidas de prevención e intervención eficaces adaptadas a contextos educativos. Tomando como objeto de estudio la etapa de Educación Secundaria Obligatoria, el principal objetivo de la investigación va a ser descubrir y constatar, mediante la opinión del alumnado, la incidencia de la violencia escolar en la provincia de Ourense. 


\section{Método}

\section{Participantes}

La muestra, quedó compuesta por 1270 alumnos, muy superior a la muestra necesaria obtenida por el programa STATS (368 alumnos) que cursan Educación Secundaria Obligatoria en Ourense durante el curso escolar 2014-15. Así pues, es suficientemente extensa y representativa para que los datos del estudio puedan ser generalizados a la población de referencia. Además, se conforma homogénea y equilibrada en términos generales: a nivel alumnado [Sexo: mujeres (620), hombres (650); Edad: 12 años (258), 13 años (291), 14 años (318), 15 años (262), 16 años (141); Expediente académico: repetidores (369), aprobados (685), con alguna materia suspensa (216); Estructura familiar: estructurada (936), desestructurada (334)]; y en referencia al centro educativo [Ubicación: rurales (754), urbanos (516); Tipo de Centro: públicos (877), concertados (393); Curso: $1^{\circ} \mathrm{ESO}(339), 2^{\circ} \mathrm{ESO}$ (380), $\left.3^{\circ} \operatorname{ESO}(286), 4^{\circ} \mathrm{ESO}(265)\right]$.

\section{Instrumentos}

Teniendo en consideración el contexto $\mathrm{y}$ en congruencia con el planteamiento, se decide emplear una metodología cuantitativa, utilizando como instrumento para la recolección de datos el cuestionario. En primer lugar, se ha elaborado y aplicado un cuestionario complementario ("ad hoc") con el objeto de conocer distintos datos sociodemográficos de los sujetos de la muestra, entre los que se encuentran: sexo, edad, titularidad y ubicación del centro educativo, estudios, curso realizado y estructura familiar. En segundo lugar, se ha utilizado el Cuestionario de Violencia Escolar Revisado, CUVE-R-ESO (ÁlvarezGarcía, Núñez, Rodríguez, Álvarez y Dobarro, 2011), que permite analizar la frecuencia de aparición de diferentes tipos de violencia escolar, protagonizado por el alumnado o el profesorado en clases. Este cuestionario está formado por 31 ítems redactados en forma de afirmaciones, que se valoran con una escala de tipo Likert. Las cinco alternativas abarcan los valores de 1 a 5, siendo: $1=$ Nunca; 2 = Pocas veces; $3=$ Algunas veces; $4=$ Muchas veces; $5=$ Siempre. La prueba aporta la visión personal del encuestado sobre las relaciones que se dan en los centros educativos, facilitando un perfil de los tipos de violencia más habituales, y de éstos con el profesorado. La fiabilidad de la prueba se calculó a través del coeficiente alfa de Cronbach. El cuestionario considerado en su totalidad ofrece un índice de fiabilidad alto $(\alpha=.94)$.

\section{Procedimiento}

Seleccionada la muestra y los cuestionarios a utilizar, se contacta con el equipo directivo y los orientadores de los centros, con el objeto de explicarles la finalidad y alcance de la investigación y proponerles su participación voluntaria. Obtenido el consentimiento de la dirección de los centros, se mantiene una reunión informativa para dar a conocer el estudio y que éstos lo trasladen a toda la comunidad educativa. Los tutores participan de forma voluntaria y no remunerada.
Paralelamente, se informa a los padres sobre la investigación y se obtiene el consentimiento de la participación de sus hijos en el estudio.

\section{Resultados}

En este estudio, se analizan cuatro factores fundamentales relacionados con la violencia en los centros educativos de la provincia de Ourense de educación secundaria obligatoria: violencia del alumnado (VA), violencia del profesorado (VP), violencia a través de las tecnologías de la información y la comunicación (VTIC) y disrupción en el aula (DA). Los resultados obtenidos muestran que las puntuaciones medias más altas se encuentran en los factores disrupción en el aula $(M=2.85)$, y violencia del alumnado $(M=2.23)$. Por el contrario, las más bajas se encuentran en el factor violencia del profesorado $(M=$ 2.1 ), y en la violencia a través de las tecnologías de la información y comunicación $(M=1.59)$ (figura 1).

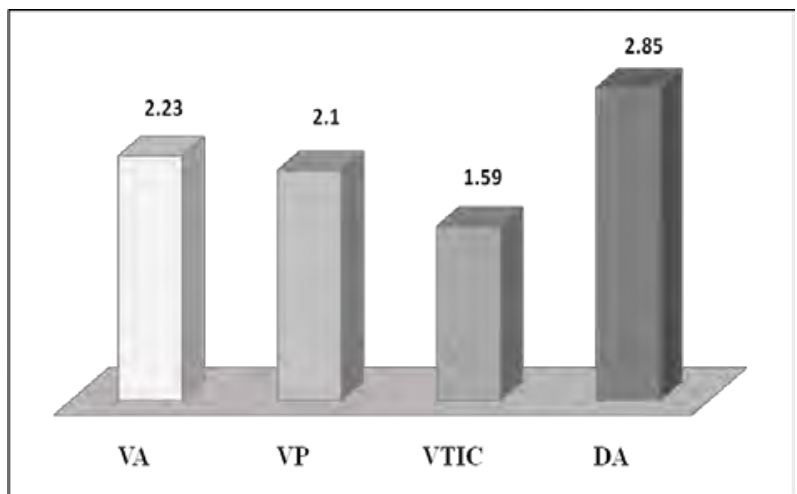

Figura 1. Factores que conforman la violencia escolar (Puntuaciones Medias)

A continuación, para una mayor claridad y facilidad en el manejo de los datos se establecen tres niveles de violencia: bajo, que agrupa los valores 1 y 2 de la escala (Nunca o Pocas veces); medio, correspondiente al valor 3 (Algunas veces); y alto, que agrupa los valores 4 y 5 de la escala (Muchas veces o siempre).

En primer lugar, se lleva a cabo un análisis descriptivo a través de los porcentajes de la totalidad de los ítems que conforman el factor violencia del alumnado (VA). De este análisis se deduce que un $64.6 \%$ de los adolescentes ourensanos consideran que el nivel de violencia escolar es bajo, un $18.4 \%$ medio, y un $17 \%$ alto. Destacar en el nivel alto, el $47 \%$ del alumnado considera que determinados estudiantes dan collejas o cachetes a sus compañeros bromeando; el $32.6 \%$ que los estudiantes hablan mal unos de otros; y un $29.2 \%$ que hay estudiantes que extienden rumores negativos acerca de compañeros. En el nivel bajo destacar que el $90.7 \%$ de los alumnos consideran que algunos estudiantes esconden pertenencias del profesorado o material del centro necesario en su trabajo, para molestarle deliberadamente; el $80.4 \%$ que los estudiantes insultan al profesorado; y un $79.7 \%$ que algunos estudiantes protagonizan agresiones físicas en las cercanías del recinto escolar. 
En segundo lugar, se procede al análisis descriptivo de los ítems que conforman el factor violencia del profesorado. Así pues, un $68.99 \%$ de los adolescentes ourensanos estiman que el nivel de violencia del profesorado es bajo, un $17.32 \%$ lo considera medio y un $13.69 \%$ alto. Señalar en el nivel alto, que un $23.3 \%$ del alumnado opina que el profesorado baja la nota a algún o alguna estudiante como castigo; el $19.6 \%$ piensa que el profesorado tiene manía a algunos alumnos o alumnas y el $17.1 \%$ considera que el profesorado castiga injustamente. En el nivel bajo, destacar que el 85.3\% de los estudiantes opinan que el profesorado insulta al alumnado, el $83.7 \%$ que el profesorado ridiculiza al alumnado y el $75.5 \%$ que el profesorado no escucha a su alumnado.

En tercer lugar, el factor violencia a través de las tecnologías de la información y comunicación muestra que el $6.57 \%$ de los adolescentes ourensanos consideran que el nivel de este tipo de violencia es alto, el 9.07\% opinan que es medio y el $84.35 \%$ que es bajo. Indicar que dentro del nivel alto el $9.6 \%$ del alumnado opina que los estudiantes insultan a profesores o profesoras, el $8.8 \%$ considera que ciertos estudiantes envían a compañeros o compañeras mensaje con el móvil de ofensa, insulto o amenaza y el 7.3\% que algunos estudiantes envían mensajes a compañeros o compañeras a través de redes sociales (Tuenti, Facebook...) de ofensa, insulto o amenaza. En el nivel bajo, el $91.4 \%$ del alumnado cree que los estudiantes publican en internet fotos o vídeos ofensivos de profesores o profesoras, el $87.5 \%$ que los estudiantes publican en internet fotos o vídeos ofensivos de compañeros o compañeras y un $86.6 \%$ que hay estudiantes que graban o hacen fotos a profesores con el móvil para burlarse de ellos.

Finalmente, se procede al análisis descriptivo de los ítems que constituyen el factor disrupción en el aula. De dicho análisis se deduce que un $31 \%$ de los estudiantes ourensanos consideran que el nivel de disrupción en el aula es alto, el $25.16 \%$ valora que es medio y un $43.85 \%$ que es bajo. Dentro del nivel alto, un $44.4 \%$ de los estudiantes cree que hay alumnado que ni trabaja ni deja trabajar al resto, un $42.6 \%$ opina que el alumnado dificulta las explicaciones del profesorado hablando durante la clases y un 35\% expone que el alumnado dificulta las explicaciones del profesor o de la profesora con su comportamiento durante la clase. En el nivel bajo, el $60.6 \%$ de los estudiantes consideran que el alumnado falta al respeto a su profesorado en el aula y un $55.1 \%$ del alumnado cree que algunos estudiantes esconden pertenencias de sus compañeros o compañeras para fastidiar.

\section{Conclusiones}

Aunque, la violencia en ámbito escolar ha sido abordada en la literatura especializada bajo diferentes conceptos: acoso, bullying, intimidación, victimización, entre otros (Vörs, 2006), en este estudio se ha decidido hablar de violencia escolar siguiendo las investigaciones de Cerezo (2004), Díaz-Aguado (2005), y Olweus (2006). En consecuencia, la violencia es entendida como una conducta intencionada con la que se causa daño o perjuicio, que en los centros educativos puede manifestar formas muy variadas, entre las que destaca la violencia física y verbal, la exclusión social, la disrupción en las aulas y la emergente violencia a través de las tecnologías de la información y comunicación (Álvarez-García, Dobarro, Álvarez, Núñez y Rodríguez, 2014).

La gran mayoría de los miembros de las comunidades educativas de los centros de la provincia de Ourense perciben un buen ambiente escolar, si bien los tipos de violencia escolar evaluados, aparecen en mayor o menor medida en estos centros educativos. Los valores que se encontraron en este estudio son bajos, similares a los obtenidos en otras investigaciones (Cerezo, 2009; Sánchez-Lacasa y Cerezo, 2010).

Asimismo, comportamientos disruptivos son la forma de violencia educativa más extendida en toda la etapa de educación secundaria obligatoria (Badía, 2005; Muñoz, Carreras y Braza, 2004). Se sostiene que existe una relación inversa entre la gravedad de la conducta violenta y su incidencia, es decir, las conductas más graves son las de menor frecuencia. De este modo, los estudios revisados muestran importantes diferencias en el porcentaje de alumnado implicado según se trate de violencia física, verbal, psicosocial o de otro tipo (Defensor del Pueblo- UNICEF, 2007).

\section{Referencias}

Álvarez-García, D., Dobarro, A., Álvarez, L., Núñez, J. C. y Rodríguez, C. (2014). La violencia escolar en los centros de educación secundaria de Asturias desde la perspectiva del alumnado. Educación XXI, 17(2), pp. 337-360.

Badía, M. M. (2005). Gravedad e importancia de los comportamientos de indisciplina. Causas y factores asociados a ciertas conductas: una revisión de diferentes estudios. Psicología Educativa, 2(11), pp. 65-78.

Cerezo, F. (2004). La violencia en las aulas. Análisis y propuestas de Intervención. España: Pirámide.

Cerezo, F. (2009). Analyzing Bullying in Spanish School. International Journal of Psychology and Psychological Therapy, 9(3), pp. 383-394.

Defensor del Pueblo-UNICEF (2007). Violencia escolar: el maltrato entre iguales en la educación secundaria obligatoria. 1999-2006. Madrid: Publicaciones de la Oficina del Defensor del Pueblo.

Díaz-Aguado, M. J. (2005). Porqué se produce la Violencia Escolar en las Escuelas y cómo prevenirla. Revista Iberoamericana de Educación, 37, pp. 17-47.

Muñoz, J. M., Carreras, M. R. y Braza, P. (2004). Aproximación al estudio de las actitudes y estrategias de pensamiento social y su relación con los comportamientos disruptivos en el aula en la educación secundaria. Anales de Psicología, 20(1), pp. 81-91.

Olweus, D. (2006). Una revisión general. En Á. Serrano (Ed.), Acoso y Violencia en la Escuela (pp. 79-103). Madrid: Editorial Ariel. 
Sánchez-Lacasa, C. y Cerezo, F. (2010). Variables personales y sociales relacionadas con la dinámica bullying en escolares de Educación Primaria. Electronic Journal of Research in Educational Psychology, 8(3), pp. 1015-1032.
Vörs, W. (2006). Bullying, el acoso escolar. Buenos Aires: Oniro. 